Maurus Reinkowski und Renate Würsch

\title{
100 Jahre Seminar für Nahoststudien in Basel
}

https://doi.org/10.1515/asia-2019-0007

\section{Ad multos annos - 100 years of the Basel Institute for Middle Eastern Studies}

\begin{abstract}
In 2019, the Basel Institute for Middle Eastern Studies will celebrate its centennial, but the preoccupation of some Basel (and Swiss) scholars with topics of Islamic and Oriental studies dates back to the $16^{\text {th }}$ century. This paper first looks at specialists with a Basel background before the institute was founded in 1919 (Hieronymus Harder, Johann Ludwig Frey, Albert Socin, Adam Mez, Wilhelm Sarasin-His). It then presents an outline of the last hundred years of institutional Middle Eastern studies in Basel, and finishes with some reflections on the recent developments of a discipline which has undergone major changes since the 1980s in the wake of the debate on "Orientalism".
\end{abstract}

Keywords: Oriental Studies, Islamic Studies, Middle Eastern Studies, University of Basel, History of Science

Schlüsselwörter: Orientalistik, Islamwissenschaft, Nahoststudien, Universität Basel, Wissenschaftsgeschichte

Die Geschichte der Basler Nahoststudien ist bereits in zwei Publikationen behandelt worden, 1994 von Gregor Schoeler und Gudrun Schubert anlässlich des 75jährigen Bestehens des Orientalischen Seminars, ${ }^{1}$ und 2002 noch einmal von Gregor Schoeler im Rahmen eines Symposiums zur Sprachwissenschaft in Basel 1874-1999. ${ }^{2}$ Die folgenden Ausführungen verstehen sich als Ergänzung hierzu.

Das Orientalische Seminar der Universität Basel ist 1919 gegründet worden, doch reicht die Geschichte der Basler Studien orientalischer Sprachen und Kulturen - vor allem des Arabischen - um einiges weiter zurück.

Renate Würsch verfasste die Abschnitte zur historischen Entwicklung des Seminars; Maurus Reinkowski steuerte das Kapitel «üngste Entwicklungen seit 2010» bei.

1 Schoeler/Schubert 1994: 1409-1413.

2 Schoeler 2002b: 72-82.

Maurus Reinkowski: E-mail: maurus.reinkowski@unibas.ch, Renate Würsch:

E-mail: renate.wuersch@unibas.ch, Gesellschaftswissenschaften, Seminar für Nahoststudien, Universität Basel, Maiengasse 51, CH-4056 Basel, Schweiz 
Wie in anderen Ländern Europas wurden auch in der Schweiz Orientstudien bis ins 19. Jahrhundert hinein fast gänzlich im Rahmen der Theologie betrieben. Dennoch entstanden in diesem Umfeld Werke, die auch für die Geschichte der europäischen Arabistik von grosser Bedeutung waren, wie z.B. der erste Druck der lateinischen Koranübersetzung von Robert von Ketton 1543 bei Johannes Oporin in Basel, den Theodor Bibliander (um 1504-1564) veranlasste, oder die Studien Johann Heinrich Hottingers (1620-1667), der wohl der Schweizer Orientalist des 17. Jahrhunderts mit den breitesten philologischen Interessen war und neben Hebräisch auch gründlich das Arabische und das Syrisch-Aramäische studiert hatte. Mehrere Werke Hottingers wie etwa das Smegma orientale (Heidelberg 1658) bezeugen eindrücklich seine umfassende Kenntnis der semitischen Sprachen. 1667, kurz vor seinem frühen Tod durch einen tragischen Unfall, hatte Hottinger einen Ruf nach Leiden auf den Lehrstuhl seines im selben Jahr verstorbenen Lehrers Jacob Golius angenommen. ${ }^{3}$

Ein jüngerer Zeitgenosse Hottingers war Hieronymus Harder (1648-1675), Sohn des Basler Universitätsnotars und Stadtschreibers Johann Conrad Harder. Hieronymus Harder war, wie aus Dokumenten über die Familie Harder hervorgeht, nach Leiden berufen, „zuerst als Professor honorarius, dann als ordinarius in den morgenländischen Sprachen; ehe er diese Stelle annahm, wollte er zuvor eine Reise in Arabien und Persien vornehmen, kam nach Constantinopel, wo er an einem hitzigen Fieber gestorben; seine Manuscripten soll die öffentliche Bibliothek in Basel erhalten haben“. ${ }^{4}$ In der Tat schenkte Johann Conrad Harder der Basler Universitätsbibliothek die orientalischen Handschriften im Besitz seines Sohnes und seinen übrigen orientalistischen Nachlass. ${ }^{5}$ Offenbar hatte es auch Hieronymus Harder, wie vor ihm Heinrich Hottinger, nach Leiden gezogen, wo es - anders als in Basel ein günstiges Umfeld für professionell betriebene Orientforschung gab.

Der erste, der sich in Basel mit gründlichen - vor allem arabistischen - Studien befasste, war der Theologe und Historiker Johann Ludwig Frey (1682-1759). Freys Laufbahn als Orientalist hat Kim Sitzler 1997 in der Jubiläumsschrift zum 250jähringen Bestehen des Frey-Grynaeischen Instituts eine eingehende Untersuchung gewidmet. ${ }^{6}$ Frey unterrichtete als erster Professor in Basel an der Universität „orientalische Sprachen“. 7 Im Catalogus Professorum Academiae Basiliensis findet sich von 1706-1712 unter seinem Namen der Eintrag: „studiosam juventutem docet

3 Zu Hottinger vgl. die Überblicksartikel von Zeller 2007: 490-491 und Pfister 1972: 656-657.

4 UB Basel, Sign. AA II 2 : 11, fol. 3r.

5 Geschenkbuch der Universitätsbibliothek Basel 1692-1762, Sign. AN II 26, fol. 1r.

6 Sitzler 1997: 243-258.

7 Sitzler 1997: 255. 
Cat[h]echetica, Orientaliumque Linguarum amatores Arabica, Samaritana, etc. “8 Nach 1712 werden die orientalischen Sprachen nicht mehr erwähnt - vermutlich deshalb, weil Frey 1711 Ordinarius für Geschichte und Extraordinarius für Theologie geworden war, beides ranghöhere Fächer als die Orientalistik.

Da er in Basel keinen Lehrer fand, dürfte Johann Ludwig Frey das Arabische im wesentlichen autodidaktisch erlernt haben. Er beherrschte die Sprache aber offenbar so gut, dass er als Ansprechperson dienen konnte, als zwei syrische Christen namens Yūḥannā 'Abdallāh Fahd und Yūsuf Ni'matallāh in den Jahren 1734-1736 auf einer „fund-raising tour“9 durch die Schweiz u.a. auch Basel besuchten. Im historischen Bestand der Basler Universitätsbibliothek haben sich vier arabische Briefe erhalten, die die beiden an Frey und dessen Freund, Johannes Grynaeus, schrieben, ${ }^{10}$ sowie der Entwurf eines arabischen Briefes von Frey an [Yū] ḥannā 'Abdallāh Fahd. ${ }^{11}$

Nicht Basel war jedoch die erste Stadt in der Schweiz, in der entscheidende Schritte zu einer Verselbständigung der Arabistik als universitäre Disziplin und ihrer Loslösung von der Theologie unternommen wurden, sondern Genf. Dort wurde 1820 ein akademischer Lehrstuhl „pour l'enseignement spécial de la langue arabe“ geschaffen, den als erster Jean [eigentlich Jean-Pierre-Louis] Humbert (1792-1851), ein Schüler Silvestre de Sacys, einnahm. ${ }^{12} 1858$ folgte Bern mit der Einrichtung einer Honorarprofessur „der orientalischen Sprachen und Literatur“ für Aloys Sprenger (1813-1893). ${ }^{13}$

In Basel dauerte es bis zum Sommersemester 1871, als erstmals das Arabische im Verzeichnis der Veranstaltungen der „Philologisch-historischen Abtheilung der Philosophischen Facultät“ erschien: Dozent war Albert Socin, der im Dezember des Vorjahres gerade von seiner ersten Orientreise nach Basel zurückgekehrt war. Neben „Hebräischer Grammatik“ bot er in jenem Semester „Altarabische Dichter“ und „Vulgärarabisch“ an. ${ }^{14}$ Die Wahl dieser beiden Themen war kein Zufall. 1867 hatte Socin in Halle mit einer Dissertation über die Gedichte des altarabischen Dichters 'Alqama promoviert, ${ }^{15}$ über Expertise in „Vulgärarabisch“ verfügte er durch seine zweijährige Studien- und Forschungsreise in den Vorderen Orient (1868-1870).

8 UB Basel VB Mscr O 11e (Bd. 7) S. 53. 55. 57. 59. 61 und VB Mscr O 11f (Bd. 8) S. 21.

9 Sitzler 1997: 254.

10 Frey-Gryn Mscr III 17: Nr. 130-133.

11 Frey-Gryn Mscr VI 9, 295r-297v.

12 Louca 1970: 19.

$13 \mathrm{Zu}$ Sprengers ungemein wechselvoller, z.T. auch abenteuerlicher Karriere, die ihn u.a. nach Indien, an den Hof des Königs von Oudh, führte, vgl. Mantl 1993.

14 Verzeichniss der Vorlesungen an der Universität Basel im Sommer-Semester 1871, Sign.: UB Basel, HPaed b III 4: 1862-1891, S. 7.

15 De 'Alkamae El Faḥl carminibus et vita. Adjecto textu Arabico adhuc inedito. 
Albert Socin, geboren am 13. Oktober 1844 in Basel, zählt gewiss zu den bedeutendsten deutschsprachigen Orientalisten des 19. Jahrhunderts. Obwohl er 1873 an der Basler Universität zum ausserordentlichen Professor ernannt wurde, war aber die Zeit für eine feste Etablierung der Arabistik in Basel noch nicht gekommen. Ende 1875 wurde Socin von Basel nach Tübingen wegberufen - als Ordinarius der semitischen Sprachen. Die Krönung seiner akademischen Karriere bildete 1890 die Berufung nach Leipzig auf den Lehrstuhl seines zwei Jahre zuvor verstorbenen Lehrers Heinrich Fleischer. In Leipzig lebte und lehrte Socin bis zu seinem Tod am 24. Juni 1899. ${ }^{16}$

Den Weg für institutionell etablierte Orientstudien an der Universität Basel bereitete Adam Mez (1869-1917). Mez, aus Freiburg i.Br. stammend, hatte 1892 in Strassburg bei Theodor Nöldeke mit einer Dissertation über die Geschichte der mesopotamischen Stadt Harrān promoviert und habilitierte sich 1894 in Basel. 1897 folgte die ausserordentliche, 1905 die ordentliche Professur. ${ }^{17} 1914$ brach der Erste Weltkrieg aus. Adam Mez wurde als deutscher Staatsangehöriger zum Kriegsdienst eingezogen. Damit der Lehrbetrieb aufrechterhalten werden konnte, wurde ein Fachkollege von Mez, Friedrich Schulthess, seit 1914 in Strassburg tätig, nach Basel verpflichtet. Im Frühjahr 1917 erhielt Schulthess einen Lehrauftrag für „orientalische Sprachen“ mit der Auflage, sich mit Prof. Mez, „falls er wieder hier sein wird“ über die Abhaltung von Vorlesungen und Übungen zu verständigen, wobei Mez das Vorbestimmungsrecht zugestanden wurde. ${ }^{18}$ Kurz nach seiner Rückkehr nach Basel starb Adam Mez jedoch am 29. Dezember 1917. In seiner Würdigung des Verstorbenen bedauert Mez' Universitätskollege, der Indogermanist Jacob Wackernagel (1853-1938), dass Mez, erst durch Krankheit, dann durch Kriegsdienst eingeschränkt, verhältnismässig wenig publizierte, und äussert dann die geradezu prophetischen Worte: „Freilich dürfen wir hoffen, dass das, was er in langen Jahren ausgedacht und gefunden hat, an die Oeffentlichkeit gelangen wird: die wunderbaren Schilderungen, die er, grossen Vorbildern folgend, von der Kultur der islamischen Welt zu geben vermochte. Es wird dann, dessen dürfen wir uns freuen, die gelehrte Welt erkennen, was sie an dem Dahingeschiedenen besass und es nicht wusste“. ${ }^{19}$ In der Tat erschien Mez’ magnum opus Die Renaissance des Islâms, eine umfassende Darstellung der islamischen Geschichte und Kultur im 10. Jahrhundert, postum 1922, herausgegeben

$16 \mathrm{Zu}$ Socins beeindruckendem wissenschaftlichen Werk vgl. Schoeler 2002b: 73-75; Würsch 2006: 89-104.

17 Staatsarchiv Basel-Stadt, UA XI 3, 1 (1900-1912), Brief des Erziehungsdepartements an die Regenz der Unversität, 17. Juli 1905.

18 Staatsarchiv Basel-Stadt, UA XI 3, 1 (1917-1920), Brief des Erziehungsdepartements an das Universitätsrektorat, 19. Februar 1917.

19 Zum Gedächtnis an Prof. Dr. Adam Mez, geboren 8. April 1869, gestorben 29. Dezember 1917; UB Basel, Sign. Fz 251:1917 Mez. 


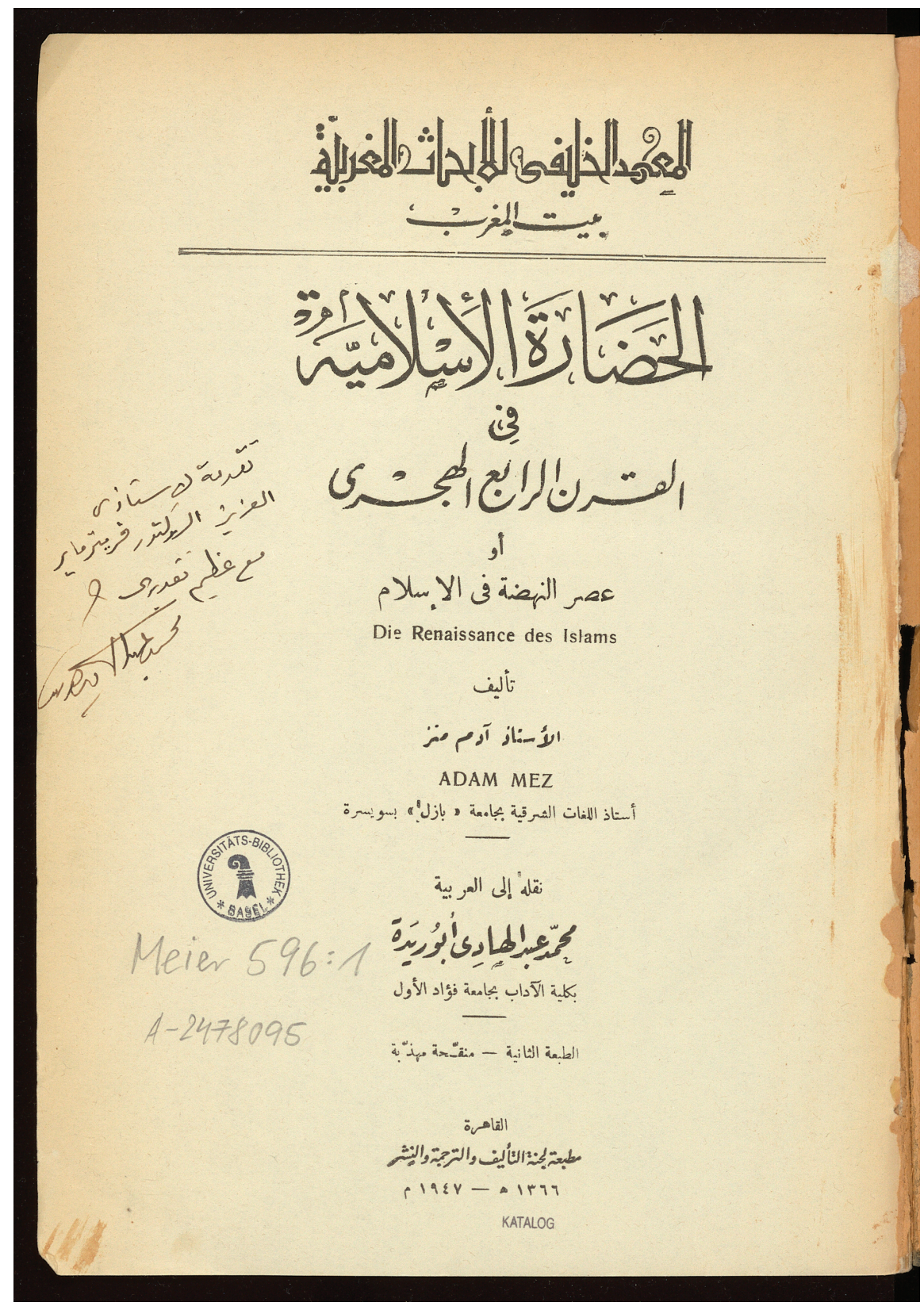

Titelblatt der arabischen Übersetzung der Renaissance des Islâms von Adam Mez mit persönlicher Widmung des Übersetzers, Muḥammad 'Abd al-Hādī Abū Rĩda, an Fritz Meier (UB Basel, Sign. Meier 596:1) 
von Hermann Reckendorf. Das Werk wurde ins Arabische, Englische, Polnische, Russische und Spanische übersetzt und wird bis heute rezipiert. ${ }^{20}$

Die durch den Ausbruch des Ersten Weltkriegs bedingte Zäsur dürfte auch der Grund dafür gewesen sein, dass ein Basler Schüler von Adam Mez, Wilhelm Sarasin-His (1882-1950), der 1907 bei Mez promoviert und danach auch die venia legendi in Basel erworben hatte, freiwillig darauf verzichtete, an der Universität zu lehren. In einem auf den 12. Dezember 1914 datierten Brief ${ }^{21}$ informierte Sarasin den damaligen Dekan der philosophischen Fakultät, Hans Rupe, über seinen Rücktritt wegen „der aussergewöhnlichen Verhältnisse, in denen wir gegenwärtig leben“ und kündigte an, sich einer Tätigkeit im Betrieb seines Vaters [d.h. des Bandfabrikanten Wilhelm Sarasin, 1855-1929] zuzuwenden. Wahrscheinlich haben neben den Zeitläuften auch die fehlenden Aussichten auf eine besoldete Stelle als Orientalist an der Universität Basel Sarasins Entscheidung mitbeeinflusst.

Friedrich Schulthess (1868-1922), der nach dem Tod von Adam Mez allein das Fach der Orientalistik in Basel vertrat, stammte aus einer alteingesessenen Zürcher Familie $^{22}$ und hatte zuerst in Basel, Göttingen und Zürich Theologie studiert. ${ }^{23}$ In Göttingen war er durch Paul de Lagarde erstmals in Kontakt zur Orientalistik gekommen und war nach dessen Tod 1891 nach Strassburg gegangen, um bei Theodor Nöldeke weiterzustudieren. 1894 promovierte er bei Nöldeke. Seinem Basler Lehrer der Theologie, Franz Overbeck, übersandte Schulthess „als ehemaliger Hörer" seine Dissertation (Probe einer syrischen Version der Vita St. Antonii. Leipzig 1894) mit den Worten: ,...bevor ich meine theologischen Kenntnisse ganz vergesse und blosser Orientalist werde, würde ich gerne noch einmal ein Grenzgebiet streifen; ich besitze nun zufällig seit mehreren Jahren Euseb's Theophanie, ed. Lee, von welcher der Herausgeber selbst s.Z. eine vielfach fehlerhafte engl. Uebersetzung veröffentlicht hat ${ }^{24}$... Würde sich eine neue Uebersetzung wohl lohnen?“25 - Wie auch immer Overbecks Antwort ausgefallen ist - das Vorhaben hat Schulthess nie verwirklicht. Vielsagend ist die Selbstbezeichnung „blosser Orientalist“. Sie ist als später Reflex der althergebrachten Hierarchie zu deuten: das vornehmste Fach war auch zu Schulthess' Zeit immer noch die Theologie.

1895 habilitierte sich Schulthess in Göttingen, wurde fünf Jahre später dort zum Titularprofessor ernannt und 1910 als Ordinarius nach Königsberg berufen.

20 So etwa jüngst bei Bauer 2018: 38, 42.

21 Staatsarchiv Basel-Stadt, UA XI 3,1 (1913-1916).

22 Schulthess 1908: Tafel IX (Schulthess zu Hottingen).

23 Vgl. das Vorwort in Schulthess' postum 1922 erschienener Publikation Die Machtmittel des Islams, III.

24 I.e. On the Theophania or Divine Manifestation of Our Lord and Saviour Jesus Christ, erschienen 1843 in Cambridge.

25 UB Basel, NL 53 B I 314. 
In Königsberg blieb er bis 1914, wechselte dann zunächst nach Strassburg als Nachfolger des nach Göttingen berufenen Enno Littmann und schliesslich 1917 nach Basel. ${ }^{26}$

Nachdem sich Schulthess in Basel fest etabliert hatte, setzte er sich offenbar nachdrücklich für die Gründung eines Instituts für sein Fach ein. Zu Beginn des Jahres 1919 erklärte sich die Kuratel der Universität „mit der Schaffung einer semitischen Abteilung des indogermanischen Seminars“ einverstanden, wollte dafür aber keine besonderen Räumlichkeiten zur Verfügung stellen, sondern schlug vor, dieses neu geschaffene Seminar solle mit dem Indogermanischen Seminar die Räumlichkeiten teilen. ${ }^{27}$ Dieser Vorschlag fand offenbar Gehör, denn im Jahresbericht für das Jahr 1919 konnte Schulthess vermelden, dass das Seminar auf das Sommersemester 1919 gegründet worden sei. Der Bücherbestand betrug „28 Werke in 30 Bänden““. ${ }^{28}$ Erstmals im Vorlesungsverzeichnis der Universität Basel erwähnt wurde das „Orientalische Seminar“ im Wintersemester 1919/20. Jacob Wackernagel, Vorsteher des damals seit neun Jahren bestehenden Indogermanischen Seminars, gewährte dem neu gegründeten Institut Hausrecht im Domizil der Indogermanisten am Stapfelberg 9. Bis zum Sommersemester 1932 sollte das Orientalische Seminar dort verbleiben. ${ }^{29}$

Friedrich Schulthess war in erster Linie Sprachwissenschaftler, arbeitete zur arabischen Dichtung - er edierte die Diwane der altarabischen Dichter Hātim aț-Tầ'ì und Umayya b. abī ș-Ṣalt - und machte sich vor allem als Erforscher der aramäischen Dialekte einen Namen. ${ }^{30} \mathrm{Zu}$ seinen aramaistischen Forschungsbeiträgen gehört auch die Edition und deutsche Übersetzung der älteren syrischen Version ${ }^{31}$ des letztlich auf das indische Pañcatantra zurückgehenden Fürstenspiegels Kalīla wa-Dimna. ${ }^{32}$ In seiner Rezension schreibt Theodor Nöldeke anerkennend über seinen früheren Schüler: „...er hat erfüllt, was man von einem so tüchtigen Kenner der

26 Schulthess 1922: IV. Zu Schulthess’ Berufung nach Königsberg vgl. Tilitzki 2012: 309-310.

27 Brief der Kuratel an die philologisch-historische Abteilung der philosophischen Fakultät, 3. Januar 1919.

28 Staatsarchiv Basel-Stadt, CC 1p (1914-1941).

29 Danach folgten mehrere Umzüge. Von 1932-1940 befand sich das Orientalische Seminar am Rheinsprung 21, von 1940-1942 am Münsterplatz 19, von 1943-1970 am Rheinsprung 20, von 1970-1993 am Hirschgässlein 21 und von 1993-2008 an der Missionsstrasse 64A. Der bisher letzte Umzug fand 2008 statt, an die Maiengasse 51.

30 Vgl. dazu Mathys 2002: 65-67.

31 Dazu Riedel 2011: 386-394.

32 Kalila und Dimna, erschienen 1911 in Berlin, ist 100 Jahre später von De Gruyter wieder aufgelegt worden. 
Sprache und exakten Philologen verlangen konnte. Besser hätte es nicht leicht einer gemacht.“33

Mit welcher philologischen Akribie Schulthess arbeitete, und wie methodisch er dabei vorging, zeigt seine auch kulturvergleichend interessante Untersuchung Zurufe an Tiere im Arabischen. ${ }^{34}$ Obwohl er offenbar nicht auf Resultate eigener Feldforschung zurückgreifen konnte - er regt solche im Vorwort der Untersuchung an - erschloss Schulthess mit dieser Studie der arabischen Lexikographie und Dialektologie ein reizvolles neues Spezialgebiet.

Am 4. Januar 1922 starb Friedrich Schulthess mitten aus seiner Tätigkeit heraus im Alter von erst 54 Jahren. Offenbar war es keine Frage, das erst zweieinhalb Jahre bestehende Fach der Orientalistik an der Universität Basel weiter bestehen zu lassen, zumal mit Rudolf Tschudi (1884-1960) ein tüchtiger junger Forscher bereit war, die Nachfolge zu übernehmen. Tschudi, in Glarus geboren, aber in Basel aufgewachsen, hatte sein Studium 1904 in Basel begonnen, mit Georg Jacob (1862-1937) in Erlangen jedoch einen Lehrer gefunden, der seinen Forschungsinteressen in idealer Weise entgegenkam. 1910 promovierte Tschudi bei Jacob mit einer Dissertation über das Āṣafnāme des Luṭī Pascha. Nach der Promotion wurde er Assistent von Carl Heinrich Becker am Seminar für Geschichte und Kultur des Hamburger Kolonialinstituts. 1911 übersiedelte er nach Tübingen, um sich dort auf die Habilitation vorzubereiten. Als der Erste Weltkrieg ausbrach, wurde Tschudi jedoch, noch bevor er sich habilitiert hatte, ans Hamburger Kolonialinstitut berufen, als Nachfolger Beckers, der 1913 einem Ruf nach Bonn gefolgt war. Nach dem Krieg kehrte Tschudi in die Schweiz zurück, zunächst nach Zürich als ausserordentlicher Professor für orientalische Sprachen, dann nach Basel als Nachfolger von Friedrich Schulthess. Bis 1949 leitete Rudolf Tschudi die Geschicke des Basler Orientalischen Seminars. Obwohl er 1929 einen Ruf nach Göttingen erhielt, blieb er Basel treu. ${ }^{35}$

Tschudis hauptsächliches Arbeitsgebiet war die osmanisch-türkische Sprache und Kultur sowie die Geschichte des Osmanischen Reiches im Wechselspiel der zeitgenössischen politischen Kräfte. Sein wissenschaftliches Profil prägte auch das Lehrangebot des Basler Orientalischen Seminars: Neben das Arabische trat nun auch das Türkische - und die dritte Hauptsprache der islamischen Kulturgeschichte, das Neupersische. Dagegen löste sich das Seminar von der Semitistik, die in der Folge wieder näher zur theologischen Fakultät (Lehrstuhl Altes Testament) rückte.

33 Nöldeke 1911: 578.

34 Schulthess 1912. Postum erschien die Ergänzung: „Noch einige Zurufe an Tiere“; Schulthess 1923: 14-19.

$35 \mathrm{Zu}$ Tschudis wissenschaftlichem Werdegang vgl. Taeschner 1961: 4-5 und Meier 1962: 138-141. 
Von der immensen Korrespondenz, die Tschudi mit Fachkollegen führte man kann durchaus von einem internationalen Netzwerk sprechen - legen die in seinem Nachlass erhaltenen Briefe beredtes Zeugnis ab. ${ }^{36}$ Sie sind eine unschätzbare, wissenschaftlich noch kaum ausgewertete Quelle für die Geschichte der deutschsprachigen Orientalistik in der ersten Hälfte des 20. Jahrhunderts.

Hervorzuheben ist ferner Rudolf Tschudis Sammlungstätigkeit - schon früh hatte er damit begonnen, arabische, persische und osmanisch-türkische Handschriften zu erwerben. Er pflegte eine enge Beziehung zur Basler Universitätsbibliothek, die ihm den grössten Teil ihres orientalischen Handschriftenbestandes verdankt. ${ }^{37}$ Auch setzte er sich selbstlos für universitäre Belange ein, beispielsweise als langjähriger Vorsteher der Bibliothekskommission der Universität oder in seinen erfolgreichen Bemühungen um die Einrichtung der Ägyptologie in Basel.

Tschudis wissenschaftliches Euvre ist nicht sehr breit, aber exklusiv und Ergebnis grosser Sorgfalt und hoher Ansprüche an sich selbst. Als Beispiel sei hier sein Beitrag für die Festschrift zum 70. Geburtstag seines Lehrers Georg Jacob, „Ein Schreiben Sülejmāns I. an Ferdinand I.“, 38 herausgegriffen. Er ist typisch für Tschudis wissenschaftliche Interessen: zum einen wird in dem Beitrag ein Thema aus der Geschichte des Osmanischen Reiches behandelt, zum anderen liegt ihm eine Handschrift zugrunde, ein Gebiet orientalistischer Forschung, das Tschudi zeitlebens besonders am Herzen lag. Das in der Basler Universitätsbibliothek aufbewahrte Dokument (Sign. AN V 33) ist ein Schreiben, das der osmanische Sultan Süleyman (reg. 1520-1566, in europäischen Quellen meist „der Prächtige“ genannt) Ende März/Anfang April 1535 aus Bagdad an den Habsburger Fürsten Ferdinand gerichtet hat. Tschudi verortet das Dokument zunächst in seinem historischen Kontext: Ferdinand war mit Johann Zapolya, dem Protégé Süleymans, in einen Streit um das Königreich Ungarn verwickelt und von Zapolya beschuldigt worden, für die Hinrichtung des venezianischen Abenteurers Ludovico Gritti, der in Ungarn als Bevollmächtigter Süleymans ein undurchsichtiges Spiel getrieben hatte, mitverantwortlich gewesen zu sein. Ferdinand hatte dem osmanischen Sultan mehrmals über Grittis Tod berichtet, Zapolyas Vorwurf strikt von sich gewiesen und sich überdies über Gebietsübergriffe Zapolyas beklagt. In dem vorliegenden Schreiben versichert Süleyman Ferdinand seiner Freundschaft und lässt ihn wissen, er habe Zapolya angewiesen, sich an den bestehenden Vertrag zu halten und Gebiete, die er sich unrechtmässig angeeignet habe,

36 UB Basel, Sign. NL 287.

37 Vgl. dazu Meier 1962: 138-139.

38 Tschudi 1932: 317-328. 
zurückzugeben. Tschudis Edition und deutsche Übersetzung des Dokuments bezeugen philologische Expertise, seine kodikologischen Anmerkungen grosse Erfahrung im Umgang mit Handschriften. Die Art und Weise, wie er das Dokument in die osmanisch-habsburgische Staatskorrespondenz einordnet, verrät überdies eine solide Kenntnis der einschlägigen Quellen. Der ganze Beitrag dokumentiert mustergültig Tschudis methodische Herangehensweise und seinen genauen Blick für historische Zusammenhänge.

1949 trat Rudolf Tschudi im Alter von 65 Jahren von seinem Amt zurück, um Fritz Meier (1912-1998) als Nachfolger Platz zu machen. Meiers wissenschaftliches Werk und seine Bedeutung für die Islamwissenschaft des 20. Jahrhunderts sind mehrfach ausführlich gewürdigt worden. ${ }^{39} 1937$ hatte er bei Tschudi mit einer Dissertation über den Mystiker Abū Isḥāq al-Kāzarūnī promoviert ${ }^{40} 1941$ folgte die Habilitation, eine Untersuchung über die persische Dichterin Mahsatī (12. Jahrhundert), und zugleich über die poetische Gattung des Vierzeilers, insbesondere über dessen Entstehung und Herkunft. ${ }^{41}$ Mit dem Thema seiner 1942 gehaltenen Antrittsvorlesung „Über das Wesen der islamischen Mystik“ war ein Forschungsgebiet benannt, das Meier zeitlebens beschäftigen sollte: das Phänomen religiöser Erfahrung. 1946-1948 weilte er als Gastprofessor für Persisch in Alexandria, darauf folgte das Basler Ordinariat. Bis zur Emeritierung 1982 vertrat Meier das Fach Islamwissenschaft in Basel; eine Berufung nach Harvard schlug er aus.

In seiner Lehre verzichtete Fritz Meier auf das Türkische, dafür widmete er sich dem Arabischen und Persischen in einer bis dahin in Basel noch nie vertretenen Vielfalt. Philologische Gründlichkeit bestimmte bei ihm das Studium dieser beiden Sprachen, daneben vermittelte Meier die Kenntnis ihrer klassischen Literaturen sowie der islamischen Kultur- und Religionsgeschichte und insbesondere der islamischen Mystik, des Sufismus. Auch Volkskundliches - Meier hatte auch bei Karl Meuli studiert - kam nicht zu kurz. Sozusagen alle Themen von Meiers Lehre waren auch Themen seiner Forschung, deren Ergebnisse in zahlreiche Publikationen Eingang fanden. ${ }^{42}$

Meiers intensive Quellenlektüre und Forschungstätigkeit bezeugt ferner seine grosse Sammlung unveröffentlichten Materials. Einiges davon war bereits für geplante Veröffentlichungen ausgearbeitet, anderes ist wertvolle Grundlage für

39 Umfassend von Gudrun Schubert, in: Encyclopaedia Iranica, s.v. Meier, Fritz [nur online]; Schubert 2000: 5-10. Vgl. auch van Ess 1998: 153-157; Schimmel 1999: 144-148; Landolt 2000: 143-146; Radtke 2000: 1-7; Radtke 2001: 369-376.

40 Meier 1948.

41 Meier 1963.

42 Vgl. das Schriftenverzeichnis auf der Webseite der Universitätsbibliothek Basel: https:// www.ub.unibas.ch/digi/a100/kataloge/nl-323-detailverzeichnisse/schriftenverzeichnis_meier_ fritz.pdf [abgerufen am 19.12.2018]. 
weitere Studien. So konnte zum Beispiel der zweite Band der Mahsatī, der Volksroman über Mahsatī und Amīr Aḥmad, postum noch veröffentlicht werden, ${ }^{43}$ und Tobias Nünlist wertete Meiers reiche Quellenbelege zur islamischen Dämonologie in seiner Habilitationsschrift aus. ${ }^{44}$ Von Meiers wissenschaftlichem Nachlass ${ }^{45}$ harrt der grösste Teil noch seiner Erschliessung.

Auf Fritz Meier folgte Gregor Schoeler (geb. 1944). Er hatte von 1982 bis 2009 den Lehrstuhl für Islamwissenschaft in Basel inne. Schwerpunkte seiner Forschung sind die frühislamische Überlieferung über das Leben des Propheten Mohammed, die arabische Dichtung, das Fortleben antiker Wissenschaftstradition in abbasidischer Zeit (Poetik, Rhetorik, Literaturtheorie) sowie die Kodikologie - er erfasste und beschrieb u.a. arabische Handschriften für das Verzeichnis der orientalischen Handschriften in Deutschland, VOHD.

Bedeutende Forschungsbeiträge - was sich auch an ihrer Rezeption ablesen lässt - lieferte Schoeler zur frühen islamischen Überlieferung über die Vita des Propheten $^{46}$ und das Verhältnis von Mündlichkeit und Schriftlichkeit in frühislamischer Zeit. ${ }^{47}$ Sein Interesse an der klassischen arabischen Literatur bezeugt die monumentale Edition und englische Übersetzung von Abū l-'Alā' al-Ma'arrīs (gest. 1058) Risālat al-g̈ufrān (zusammen mit Geert Jan van Gelder), 2 Bde. New York 2013-2014. Eine Vorarbeit dazu war die deutsche Übersetzung der in der Risāla enthaltenen, mit satirischen Elementen gespickten Jenseitsreise. ${ }^{48}$

Obwohl die Arabistik bei Gregor Schoeler im Vordergrund stand, hat er in der Lehre stets auch das Persische vertreten und auch im Fachbereich Iranistik publiziert. Ein Beispiel dafür ist seine auch interdisziplinär interessante Studie über die 'Lücke' in der epischen Überlieferung Irans. ${ }^{49}$ Darin erklärt Schoeler die merkwürdige Tatsache, dass in der nationalen Überlieferung Irans, insbesondere in Firdawsīs Šăhnāma, die historisch so bedeutenden Epochen der Meder, Achämeniden und Seleukiden nahezu unbekannt sind und von den Parthern (Arsakiden), die Iran immerhin rund 500 Jahre regierten, kaum mehr als ein paar Namen überliefert werden. Zur Erklärung zieht er eine Theorie von der Struktur mündlicher Überlieferung heran, die vom Ethnologen Jan Vansina entwickelt wurde. Danach ist die Vorstellung von der Vergangenheit dreifach gegliedert. Reich sind die Quellen über die Vorzeit (Ursprungsmythen und Sagen von Kulturheroen) und über die rezente Zeit (historische Quellen) - dazwischen

43 Meier 2005.

44 Nünlist 2015.

45 UB Basel, NL 323.

46 Schoeler 1996.

47 Schoeler 2002a.

48 Abū l-'Alā' al-Ma'arrī (2002).

49 Schoeler 1998: 373-392. 
befindet sich eine sich stets verschiebende Lücke (Vansina: floating gap). Grund für dieses Phänomen ist die Tatsache, dass über eine gewisse Zeittiefe hinaus die Chronologie nicht mehr gehalten werden kann, wenn sie nicht durch schriftliche Quellen gestützt wird. In der iranischen nationalen Tradition liefen, wie Schoeler nachweist, nur in der dritten, rezenten Phase mündliche und schriftliche Überlieferung nebeneinander her. Für die Zeit davor existierte nur noch eine mündliche Tradition, weil die alt- und mittelpersischen Zeugnisse nicht mehr zugänglich waren (die Kenntnis der Keilschrift und der aramäischen Schrift, in der sie abgefasst waren, war inzwischen verloren gegangen). Da die mythische Vorzeit durch eine zusätzliche (ebenfalls mündliche) religiöse Tradition geschützt war, ist sie viel besser dokumentiert als die mittlere Zeit der 'Lücke', für die nur eine säkular-nationale mündliche Überlieferung existierte.

Das Gebiet des Persischen betrifft auch Schoelers jüngstes Buch über die Rätselprinzessin Turandot, das er zusammen mit Youssef Mogtader, viele Jahre Lektor für Persisch am Basler Orientalischen Seminar, verfasst hat. ${ }^{50}$ Grundlage der Untersuchung dieses auch nach Europa gewanderten und literarisch-musikalisch adaptierten (Gozzi, Schiller, Puccini) Märchenstoffes ist ein wegweisender Aufsatz Fritz Meiers über die Turandot aus dem Jahr $1941 .{ }^{51}$ Für die Edition griffen Schoeler und Mogtader auf Kopien der persischen Handschriften der Turandoterzählung zurück, die sich in Meiers Nachlass befanden.

Eine Darstellung der Basler Arabistik des 20. Jahrhunderts wäre nicht vollständig ohne Alfred Bloch (1915-1983), der zwar nie den Lehrstuhl für orientalische Philologie bzw. Islamwissenschaft innehatte, aber als Ordinarius für Vergleichende Sprachwissenschaft mit besonderer Berücksichtigung der Indologie ${ }^{52}$ ein Nachbarfach an der Universität Basel betreute. Bloch hatte ein besonderes Interesse an der altarabischen Sprache und Dichtung. Er steuerte zu diesem Spezialgebiet zwei wichtige Publikationen bei, eine Monographie über Vers und Sprache im Altarabischen (Basel 1946) und den Aufsatz „Qașīda“53 über die zentrale - und angesehenste - Gattung der arabischen Dichtung. Blochs Beiträge zur Arabistik wurden auch von den Basler Fachorientalisten in ihrer Bedeutung gewürdigt. Als Rudolf Tschudi 1949 vom Eidgenössischen Politischen Departement - dem Vorläufer des heutigen EDA - gebeten wurde, darzulegen, was in der Basler Universität für das Studium der arabischen Sprache und Kultur getan würde - es ging um die damaligen Beziehungen zwischen der Schweiz und Ägypten - nannte er Bloch ausdrücklich und liess die Erläuterung folgen: „In den letzten Jahren hat einer

50 Schoeler 2017.

51 Meier 1941: 1-27. Nachtrag: 415-421.

52 Vgl. dazu Wachter 2002: 124-125.

53 Bloch 1948: 106-132. 
meiner jüngeren Kollegen, Herr Dr. Alfred Bloch, ... zwei arabistische Arbeiten verfasst, die bei den europäischen Fachgenossen hohe Anerkennung gefunden haben und zweifellos auch die Gelehrten in Kairo lebhaft interessieren werden. “54

\section{Jüngste Entwicklungen seit 2010}

Seit dem Jahr 2010 ist Maurus Reinkowski Professor für Islamwissenschaft am Seminar. Seine Forschungsschwerpunkte liegen auf der neueren und neuesten Geschichte des Nahen Ostens und des östlichen Mittelmeerraums. Unter allen Vorgängern kommt sein Profil dem vom Rudolf Tschudi am nächsten, der in den Jahren 1922-1949 das Seminar leitete. Klassisch islamwissenschaftliche Inhalte in Lehre und Forschung finden sich weiterhin am Seminar, prominent vertreten vor allem durch Professorin Renate Würsch.

Mit der Einführung eines neuen osmanistischen bzw. türkeikundlichen Schwerpunktes wird Türkisch seit 2010 wieder direkt am Seminar unterrichtet. Leider gelang es wegen fehlender Mittel nicht, das Persische als dritte Sprache (neben Arabisch und Türkisch) zu halten. Mit dem Herbstsemester 2013 musste der Unterricht in dieser Sprache eingestellt werden.

Drei wichtige, mit einander eng zusammenhängende Entwicklungen haben das Seminar in den 2010er Jahren geprägt. Mit der Auflösung des bi-fakultären (gemeinsam von Philosophisch-Historischer und Theologischer Fakultät getragenen) Departements Religionswissenschaften wechselte das Seminar im Jahr 2012 in das Departement Gesellschaftswissenschaften. Eine zweite Änderung war im Jahr 2013 die Umbenennung des Seminars von Orientalisches Seminar in Seminar für Nahoststudien. In einem dritten Schritt wurde 2013 das Masterstudienfach Islamwissenschaft in Near \& Middle Eastern Studies umbenannt, gefolgt von der ab 2019 wirksamen Neuausrichtung des Bachelorstudienfaches Islamwissenschaft hin zu Nahoststudien.

Das Bachelorstudienfach Nahoststudien und das Masterstudienfach Near \& Middle Eastern Studies verstehen sich als thematisch und regional ausgerichtete Studienfächer, die sich mit geschichts- und sozialwissenschaftlichen Themen des Nahen Ostens sowie mit religions- und kulturwissenschaftlichen Fragestellungen des Islams und muslimischen Lebenswelten in neuerer Geschichte und Gegenwart beschäftigen.

Die Nahoststudien beteiligen sich an zahlreichen gemeinsamen Unternehmungen des Departements Gesellschaftswissenschaften: der Graduate School for Social Sciences (G3S), dem Masterstudiengang Changing Societies und der

54 Brief vom 18. Juli 1949, UB Basel, NL 323 : F 923, 61. 
gemeinsamen sozialwissenschaftlichen Methodenausbildung auf Bachelor- und Masterebene.

Die Umbenennung des Seminars und die Neuausrichtung der Studienfächer verdanken sich der Dynamik, die sich aus der Einbettung in die Gesellschaftswissenschaften ergab. Seminarinterne Überlegungen traten hinzu: So könnte man im Begriff «Orientalisches Seminar» den Vorzug sehen, einen möglichst allgemein gehaltenen Dachbegriff für den Zusammenschluss von Fächern wie Japanologie, Sinologie, Indologie und Islamwissenschaft bereitzustellen. Eine solche Fächervielfalt im Bereich der «aussereuropäischen» Kulturen kann aber die Universität Basel nicht bieten. Zudem wollte das Seminar allgemeinen Veränderungen in der internationalen Wissenschaftslandschaft gerecht werden, in der das Begriffsfeld «Orient», «Orientalistik», «orientalisch» wegen seiner exotisierenden und zugleich abwertenden Konnotationen nicht mehr gebräuchlich ist. Gewiss ist auch der Begriff «Naher Osten» auf eurozentristische und koloniale Weltsichten zurückzuführen. Nichtsdestotrotz hat sich der Begriff in der Selbstbezeichnung der Menschen in der Region durchgesetzt, die sich selbst zwischen dem näherliegenden Westen und dem Fernen Osten verorten, so dass die Bezeichnung des «Nahen Osten» eine praktische Legitimität besitzt. Der Begriff «Nahoststudien» findet sich an mehreren deutschsprachigen Universitäten (Halle, Marburg, München, Tübingen,) sowie an einigen prominenten internationalen Universitäten (Harvard, Princeton).

Die Gesamtorientierung des Seminars für Nahoststudien in Basel weicht vom üblichen Profil islamwissenschaftlicher Standorte ab: Der thematische und zeitliche Schwerpunkt liegt in Basel auf der politischen, sozialen und intellektuellen Geschichte des spät- und post-osmanischen Nahen Ostens ab dem 19. Jahrhundert sowie in politischen und gesellschaftlichen Entwicklungen in Zeitgeschichte und Gegenwart. Geographisch gesehen liegt der Schwerpunkt auf den historischen Kerngebieten des Osmanischen Reichs, also im sogenannten «Kleineurasien», das sich zusammensetzt aus den Regionen Südosteuropa, Anatolien, Kaukasus, Levante, Mesopotamien, arabische Halbinsel und Ägypten.

Anschaulich wird diese Ausrichtung am Beispiel des in den Jahren 2013-2018 am Seminar angesiedelten Forschungsprojekts SIBA - Visuelle Zugänge zur vergleichenden Lebensweltforschung in Jugoslawien und in der Türkei. Das SIBA-Team um die SNF-Förderungsprofessorin Nataša Mišković untersuchte den Alltag ehemals osmanischer Städte (Sarajevo - Istanbul - Belgrad - Ankara) in den 1920er und 1930er Jahren. Im Mittelpunkt standen nicht schriftliche Quellen, sondern Pressefotografien aus den Archiven der grossen Tageszeitungen Jugoslawiens und der Türkei. 
Als zweites Beispiel für diesen Ansatz sei die gemeinsam mit Hans-Lukas Kieser (Universität Zürich / University of Newcastle, Australien) konzipierte Konferenzreihe «Osmanisch-türkischer Kataklysmus 1912-1922» genannt. In Basel selbst fanden in diesem Zusammenhang die beiden internationalen Tagungen The Ottoman Cataclysm: Its Beginnings (2013) und Between Realpolitik and Utopia. A Century with the Balfour Declaration (2017) statt.

Im Bereich der Ausrichtung von Konferenzen war eine weitere grössere Unternehmung des Seminars die Durchführung des 26. Kongress der Union Européenne des Arabisants et Islamisants (UEAI) 2012 in Basel. ${ }^{55}$

Das Seminar für Nahoststudien ist innerhalb der Schweiz mit den anderen islamwissenschaftlichen bzw. nahostbezogenen Standorten sehr gut vernetzt. Zu nennen ist hier die Zusammenarbeit in der Schweizerischen Asiengesellschaft und Schweizerischen Gesellschaft Mittlerer Osten und Islamische Kulturen (SGMOIK). Herauszuheben ist zudem die seit 2012 von den Universität Basel und Zürich geführte SUK-Doktoratskooperation in Islamwissenschaft und Middle Eastern Studies (MUBIT). Im Rahmen von MUBIT finden alljährlich in Basel internationale Doktorandenworkshops statt, die grossen Zuspruch gefunden haben.

Zudem hat sich in den letzten Jahren eine enge Zusammenarbeit mit dem Orientalischen Seminar der Albert-Ludwigs-Universität in Freiburg i. Br. (vertreten durch Prof. Tim Epkenhans) ergeben.

Das Seminar für Nahoststudien kämpft, wie andere vergleichbare Einrichtungen, mit dem Widerspruch zwischen einer sehr geringen Ausstattung als «Orchideenfach» und ständig wachsenden Erwartungen aus Gesellschaft, Medien und Universität an das Seminar, politische, religiöse und gesellschaftliche Entwicklungen unter Musliminnen und Muslimen zu erläutern. Das Seminar für Nahoststudien muss weiterhin einen Weg finden, einerseits diesen Ansprüchen gerecht zu werden und andererseits hohe Qualität in der Lehre und internationale Sichtbarkeit in der Forschung zu gewährleisten.

\section{Bibliographie}

Abū l-'Alā' al-Ma'arrī (2002): Paradies und Hölle. Die Jenseitsreise aus dem „Sendschreiben über die Vergebung“. Aus dem Arabischen übersetzt und herausgegeben von Gregor Schoeler. München: C.H. Beck.

Abū l'Alā' al-Ma'arrī (2013-2014): The Epistle of Forgiveness. Hrsg. und übers. von Gregor Schoeler und Geert Jan van Gelder, I-II. New York: New York University Press.

Bauer, Thomas (2018): Warum es kein islamisches Mittelalter gab. München: C.H. Beck. Bloch, Alfred (1946): Vers und Sprache im Altarabischen. Basel: Verlag für Recht und Gesellschaft.

55 Reinkowski/Winet 2016. 
Bloch, Alfred (1948): „Qașīda“. Asiatische Studien 2: 106-132.

Ess, Josef van (1998): „Fritz Meier (10.6.1912-10.6. 1998)“. In: Jahrbuch der Heidelberger Akademie der Wissenschaften für 1998. Heidelberg: Winter, 153-157.

Kalila und Dimna (1911): Syrisch und deutsch von Friedrich Schulthess. Berlin: Reimer. Landolt, Hermann (2000): „Fritz Meier [1912-1998]“. Studia Iranica 29: 143-146.

Louca, Anouar (1970): Jean Humbert, 1792-1851, arabisant genevois. Genève: Association suisse-arabe.

Mantl, Norbert (1993): Aloys Sprenger: Der Orientalist und Islamhistoriker aus Nassereith in Tirol. Zum 100. Todestag am 19. Dezember 1993. Nassereith: Selbstverlag der Gemeinde Nassereith.

Mathys, Hans-Peter (2002): Semitistik und Hebraistik. In: Die Sprachwissenschaft in Basel 1874-1999. Akten des Symposiums vom 30. Oktober 1999. Hrsg. von Rudolf Wachter. Basel: Schwabe, 61-71.

Meier, Fritz (1941): „Turandot in Persien“. Zeitschrift der Deutschen Morgenländischen Gesellschaft 95: 1-27. Nachtrag: 415-421.

Meier, Fritz (1948): Die Vita des Abū Isḥāq al-Kāzarūnī. Nach dem arabischen Grundwerk von Hațīb Imām Abū Bakr Muhammad b. 'Abdalkarīm, persisch bearb. von Maḥmūd b. 'Uțmān. Leipzig: F.A. Brockhaus.

Meier, Fritz (1962): „Rudolf Tschudi † (1884-1960)“. Der Islam 38: 138-141.

Meier, Fritz (1963): Die schöne Mahsatī. Ein Beitrag zur Geschichte des persischen Vierzeilers. Wiesbaden: Steiner.

Meier, Fritz (2005): Der Volksroman über Mahsatī und Amīr Ahmad. Hrsg. von Gudrun Schubert und Renate Würsch. Leiden: Brill.

Nöldeke, Theodor (1911): „Kalila und Dimna, Syrisch und deutsch von Friedrich Schultheß“ [Rez.] Zeitschrift der Deutschen Morgenländischen Gesellschaft 65: 578-588.

Nünlist, Tobias (2015): Dämonenglaube im Islam. Berlin: De Gruyter.

On the Theophania or Divine Manifestation of Our Lord and Saviour Jesus Christ (1843):

Translated into English with notes, from an ancient Syriac version of the Greek original now lost ... ed. by Samuel Lee. Cambridge: Duncan and Malcolm.

Pfister, Rudolf (1972): „Hottinger, Johann Heinrich“. Neue Deutsche Biographie 9: 656-657.

Radtke, Bernd (2000): „Fritz Meier (10.6.1912-10.6. 1998)“. Persica 16: 1-7.

Radtke, Bernd (2001): „Fritz Meier (10.6.1912-10.6. 1998)“. Oriens 36: 369-376.

Reinkowski, Maurus / Winet, Monika (Hrsg.) (2016): „Arabic and Islamic Studies in Europe and Beyond“. Proceedings of the 26th Congress of the Union Européenne des Arabisants et Islamisants, Basel 2012. Leuven: Peeters.

Riedel, Dagmar (2011): „Kalila wa Demna, I. Redaction and circulation“. In: Encyclopaedia Iranica. Hrsg. von Ehsan Yarshater, Vol. XV. London: Routledge and Kegan Paul, 386-394.

Schimmel, Annemarie (1999): „In memoriam Fritz Meier“. Die Welt des Islams 39(2): 144-148.

Schoeler, Gregor (1996): Charakter und Authentie der muslimischen Überlieferung über das Leben Mohammeds. Berlin: De Gruyter. Ins Englische übersetzt u.d.T.: The biography of Muhammad. Nature and authenticity. New York: Routledge, 2011.

Schoeler, Gregor (1998): „Die 'Lücke' in der nationalen Tradition Irans“. In: Annäherung an das Fremde. Hrsg. von Holger Preissler und Heidi Stein. [XXVI. Deutscher Orientalistentag vom 25. bis 29.9. in Leipzig. ZDMG. Suppl. XI]. Stuttgart: Steiner, 373-392.

Schoeler, Gregor (2002a): Écrire et transmettre dans les débuts de l'Islam. Paris: Presses universitaires de France. Ins Englische übersetzt u.d.T.: The genesis of literature in Islam. From the aural to the read. Edinburgh: Edinburgh University Press 2009. 
Schoeler, Gregor (2002b): Orientalische Philologie und Sprachwissenschaft. In: Die Sprachwissenschaft in Basel 1874-1999. Akten des Symposiums vom 30. Oktober 1999. Hrsg. von Rudolf Wachter. Basel: Schwabe, 72-82.

Schoeler, Gregor / Mogtader, Youssef (2017): Turandot, die persische Märchenerzählung. Edition, Übersetzung, Kommentar. Wiesbaden: Dr. Ludwig Reichert Verlag.

Schoeler, Gregor / Schubert, Gudrun (1994): „75 Jahre Orientalisches Seminar, 100 Jahre Islamwissenschaft an der Universität Basel“. Asiatische Studien 48: 1409-1413.

Schubert, Gudrun (2000): „Fritz Meier (1912-1998)“. Zeitschrift der Deutschen Morgenländischen Gesellschaft 150: 5-10.

Schubert, Gudrun: „Meier, Fritz“. In Encyclopaedia Iranica. Hrsg. von Ehsan Yarshater. [nur online, http://www.iranicaonline.org/articles/meier-fritz-1, abgerufen am 19.12.2018].

Schubert, Gudrun (2018): Verzeichnis der Schriften von Fritz Meier. https://www.ub.unibas.ch/ digi/a100/kataloge/nl-323-detailverzeichnisse/schriftenverzeichnis_meier_fritz.pdf (abgerufen am 12/19/2018).

Schulthess, Friedrich (1912): Zurufe an Tiere im Arabischen. Berlin: Königliche Akademie der Wissenschaften.

Schulthess, Friedrich (1922): Die Machtmittel des Islams. Zürich: Schulthess \& Co.

Schulthess, Friedrich (1923): „Noch einige Zurufe an Tiere“. Zeitschrift für Semitistik und verwandte Gebiete 2: 14-19.

Schulthess, Hans (1908): Die Familie Schulthess von Zürich. Festschrift zur Feier des einhundertundfünfzigsten Bestehens der Schulthess'schen Familienstiftung. Zürich: Schulthess \& Co.

Sitzler, Kim (1997): „Dignitas Arabicae. Johann Ludwig Frey, ein Typus der orthodox-protestantischen Arabistik“. In: Im Spannungsfeld von Gott und Welt. Beiträge zu Geschichte und Gegenwart des Frey-Grynaeischen Instituts in Basel 1747-1997. Hrsg. von Andreas Urs Sommer. Basel: Schwabe, 243-258.

Socin, Albert (1867): De 'Alḳamae El Faḥl carminibus et vita. Adjecto textu Arabico adhuc inedito. Halis Saxonum: Typis F.L. Metzgeri Lipsiensis.

Taeschner, Franz (1961): „Rudolf Tschudi (1884-1960)“. Zeitschrift der Deutschen Morgenländischen Gesellschaft 111: 4-5.

Tilitzki, Christian (2012): Die Albertus-Universität Königsberg. Berlin: Akademie-Verlag.

Tschudi, Rudolf (1932): „Ein Schreiben Sülejmāns I. an Ferdinand I“. In: Festschrift Georg Jacob zum siebzigsten Geburtstag, 26. Mai 1932. Hrsg. von Theodor Menzel. Leipzig: Harrassowitz Verlag, 317-328.

Verzeichniss der Vorlesungen an der Universität Basel im Sommer-Semester 1871. Basel: Universität.

Wachter, Rudolf (2002): „Vergleichende Sprachwissenschaft“. In: Die Sprachwissenschaft in Basel 1874-1999. Akten des Symposiums vom 30. Oktober 1999. Hrsg. von Rudolf Wachter. Basel: Schwabe, 112-129.

Würsch, Renate (2006): „Albert Socin (1844-1899)“. In: Palaestina exploranda. Studien zur Erforschung Palästinas im 19. und 20. Jahrhundert anlässlich des 125jährigen Bestehens des Deutschen Vereins zur Erforschung Palästinas. Hrsg. von Ulrich Hübner. Wiesbaden: Harrassowitz Verlag, 89-104.

Zeller, Rosmarie (2007): „Hottinger, Johann Heinrich“. In: Historisches Lexikon der Schweiz, Bd. Vol. 6. Hrsg. Marco Jorio. Basel: Schwabe, 490-491.

Zum Gedächtnis an Prof. Dr. Adam Mez, geboren 8. April 1869, gestorben 29. Dezember 1917 (1917): Basel. 